\title{
Revenus des médecins indépendants de Suisse en 2006 (nouveau) et 2005 (réévaluation)*
}

Martina Reichert

\footnotetext{
* Analyse réalisée par Martina Reichert de Sophistic Soft (Bureau de conseil spécialisé en analyses statistiques dans le système de santé, fondé par le Dr Niklaus Hasler) sur mandat de la Fédération des médecins suisses (FMH)
}

La présente analyse peut être téléchargée sur le site internet de la FMH www.fmh.ch $\rightarrow$ AUTRES THEMES $\rightarrow$ Démographie médicale $\rightarrow$ Revenus La numérotation des pages, des tableaux et des graphiques fait référence au document intégral.

L'analyse complète (disponible uniquement en allemand) est remise sur demande adressée à kommunikation@fmh.ch

Correspondance: Martina Reichert Weidenweg 4 CH-8492 Wila

\section{A. Introduction}

Le 15 octobre 2009, la Fédération des médecins suisses (FMH) a chargé le bureau Sophistic Soft d'analyser les revenus des médecins indépendants de Suisse pour l'année fiscale 2006 par un nouveau recensement complet. Le Dr Niklaus Hasler-Gloor, fondateur et longtemps seul auteur de cette analyse, est décédé le 9 septembre 2009. Sophistic Soft reprend le flambeau en poursuivant le travail précurseur fourni par Niklaus Hasler. A l'instigation du Département «Données, démographie et qualité $\mathrm{DDQ}$ » de la $\mathrm{FMH}$, quelques critères supplémentaires importants ont été pris en compte dans les évaluations, dont notamment:

- la répartition par sexe dans différents tableaux

- la représentation synoptique des valeurs moyennes et centrales

- l'intégration de la valeur centrale dans presque tous les tableaux

- l'indication de la valeur maximale dans presque tous les tableaux

- des tableaux croisés supplémentaires concernant le canton, la spécialisation et le sexe, la comparaison détaillée de la statistique médicale et des types de données évaluées

- l'intégration de boxplots dans les graphiques

La présente analyse fait directement suite aux 25 premiers rapports établis entre août 1977 et août 2008.

Le chapitre A est consacré à des Remarques générales sur les jalons historiques durant la période considérée, sur la base de données et leur représentativité ainsi que sur les méthodes et principes, tandis que le chapitre B présente un Résumé des principaux résultats, tableaux et graphiques. Ce résumé se limite aux chiffres concernant les médecins âgés de moins de 66 ans. La version intégrale, en revanche, publie également les chiffres concernant les médecins âgés de plus de 66 ans et les chiffres concernant l'ensemble des médecins (indépendamment de l'âge).

Par ailleurs, la version complète contient une analyse approfondie d'autres critères tels que la dépendance du revenu par rapport à la somme salariale du personnel, à l'âge du médecin ou au nombre d'années en cabinet privé.

La numérotation des tableaux ainsi que les renvois aux différents chapitres et à la pagination font référence à la version intégrale de l'étude.

\section{A.1 Jalons historiques}

Dans les tableaux indiquant une évolution historique, les années du siècle dernier sont regroupées par groupes de deux. A l'époque, les revenus n'étaient recensés que tous les deux ans (au rythme des périodes fiscales de l'impôt fédéral direct). Avec l'introduction en 2001 du système d'imposition postnumerando, la taxation des cotisations AVS des travailleurs indépendants est passée du système d'imposition sur la base du revenu antérieur (praenumerando) au système d'imposition sur la base du revenu actuel (postnumerando). Tombant dans la brèche de calcul, les revenus professionnels des années 1999 et 2000 n'ont pu être ni recensés ni analysés, d'où l'absence de ces deux années dans les tableaux et les graphiques qui indiquent une évolution dans le temps. C'est le cas par exemple du Tableau 23 illustrant l'évolution de différents indices depuis 1971. Pour des raisons de place, seule la troisième partie (depuis 1995/96) a été reproduite.

En 2005, l'Office fédéral de la statistique (OFS) a abandonné la distinction entre travailleurs et employés. Depuis 1993, les données sont réparties par secteurs d'activité. La présente analyse recourt également à cette distinction à partir de 1995/96 en reprenant toutefois les indices de 1993 (travailleurs = secteur II, employés = secteur III), même si ces catégories ne correspondent pas exactement à l'ancienne classification.

L'introduction du nouveau tarif TARMED au $1^{\text {er }}$ janvier 2004 avait comme ambition de réduire entre autres la dispersion des revenus des différentes spécialisations. Tout comme le rapport de novembre 2008, la présente analyse des revenus 2005 et 2006 tient uniquement compte des revenus réalisés après l'introduction du TARMED. Les chiffres de 2006 permettent désormais de disposer des résultats de trois années complètes de facturation selon le TARMED.

\section{A.2 Base de données et représentativité}

Les données qui constituent la base de l'analyse de Sophistic Soft proviennent notamment des sources suivantes:

- medisuisse: comme la Caisse de compensation AVS des médecins, dentistes, vétérinaires et chiropraticiens de St-Gall facture des frais administratifs inférieurs à d'autres caisses de compensation, la grande majorité des médecins indépendants 
versent leurs propres cotisations AVS ainsi que celles de leur personnel à medisuisse. La part des médecins affiliés à medisuisse varie selon le canton, mais s'élève à $75 \%$ à l'échelle nationale. La grande majorité des analyses présentées par Sophistic Soft se base sur les données qui lui ont été transmises par medisuisse. En août 2009, les cotisations AVS de 12730 médecins (9643 hommes, 3087 femmes) ont fait l'objet d'une décision définitive pour l'année d'imposition 2005, les cotisations de 11853 médecins (8917 hommes, 2936 femmes) pour l'année d'imposition 2006;

- Fédération des médecins suisses (FMH): la FMH met à la disposition de Sophistic Soft un grand nombre de données. Nous remercions ici Martina Hersperger et Esther Kraft du Département Données, démographie et qualité de leur précieuse coopération, notamment concernant la préparation et l'adaptation des statistiques médicales;

- Office fédéral de la statistique (OFS): relevé de la structure salariale, indice suisse des prix à la consommation;

- autres sources: Office fédéral des assurances sociales, santésuisse, etc.

Pour clarifier la représentativité de l'étude, la présente analyse commence pour la première fois par des tableaux indiquant la part des données connues et traitées par rapport à l'ensemble du groupe concerné. Ces tableaux montrent les sous-groupes (cantons, spécialisations, sexe) ayant fait l'objet d'une attention toute particulière dans l'interprétation (cf. aussi paragraphe suivant). La comparaison entre le nombre de données fournies par medisuisse et celui des médecins saisis dans la statistique médicale de la FMH montre que l'étude peut revendiquer une représentativité exceptionnelle grâce à l'analyse de près des trois-quarts de toutes les valeurs individuelles. Afin d'améliorer la représentativité de certains groupes (notamment des cantons et spécialisations, à l'avenir peut-être aussi des combinaisons de paramètres), il serait souhaitable de pouvoir évaluer les données de tous les médecins indépendants.

\section{A.3 Méthodes et principes}

Un relevé statistique présente des valeurs numériques sensées se rapprocher le plus possible de la valeur effective de l'échantillon. En absolu, le résultat correct ne peut être calculé ou déterminé que si les données de tous les individus de l'échantillon considéré sont connues et disponibles pour l'évaluation.

La représentativité statistique d'un groupe de données implique un nombre minimal de 50 valeurs individuelles. Plus le groupe est important, plus les résultats se rapprochent des chiffres effectifs en tenant compte de toutes les valeurs individuelles. A condition néanmoins que les données à évaluer soient des données normalement réparties et que les sujets aient été choisis au hasard.
La présente étude ne remplit aucune de ces deux conditions. Les revenus des médecins ne sont pas normalement répartis, mais présentent une dissymétrie positive (la moyenne est supérieure à la valeur centrale). En outre, les sujets ne sont pas choisis au hasard, mais correspondent à la part des médecins affiliés à medisuisse.

Le rôle de ce présent rapport est de renseigner sur les revenus des médecins, à la fois de l'ensemble des médecins en cabinet privé mais aussi de certains groupes du corps médical, classés selon des critères tels que la spécialisation, le canton, le sexe ou l'âge. Sophistic Soft a décidé de ne prendre en compte dans l'évaluation que les sous-groupes présentant plus de 20 valeurs individuelles, bien que la limite inférieure d'un groupe ait été fixée à 50 , comme mentionné plus haut. Le Tableau 1c qui renseigne sur le nombre de médecins par canton et spécialisation révèle par exemple qu'un quart seulement des quantités partielles satisfait à l'exigence $\mathrm{N}>20$. C'est pourquoi l'évaluation des spécialisations ne prend pas en considération la pathologie anatomique ni la médecine tropicale. Les autres résultats sont publiés sous réserve expresse d'une certaine prudence dans l'interprétation des données correspondant à la valeur critique de N.

Par ailleurs, les groupes pour lesquels Sophistic Soft disposait de données pour moins de 30\% des médecins établis selon la statistique médicale, ont été ignorés. Ceci concerne notamment les médecins du canton de Genève dont $15 \%$ seulement sont affiliés à medisuisse. La raison de cet écart remarquable reste inconnue.

Toutefois, le calcul des valeurs de groupes plus importants (par ex. toute la Suisse ou tous les internes) a toujours pris en compte l'intégralité des données.

L'une des observations intéressantes faites pour les valeurs normalement réparties porte sans aucun doute sur la dispersion des revenus, reflétée par la valeur de l'écart type. Pour les valeurs non normalement réparties telles que les revenus des médecins, la valeur quelque peu abstraite de la dissymétrie serait toutefois plus pertinente. Afin de faciliter la lecture et la compréhension de la dispersion des revenus, on a préféré compléter les séries de chiffres avec la valeur maximale. Dans presque chaque groupe, la valeur minimale des revenus des médecins correspond à 0 .

Comme la représentation synoptique des valeurs moyenne et centrale ainsi que l'intégration de la valeur maximale ont élargi les tableaux, nous renoncerons dans ce résumé à indiquer les valeurs réévaluées de 2005 pour nous limiter à reproduire les nouvelles données recensées pour 2006 ainsi que l'écart par rapport à 2005 des valeurs centrale et moyenne.

Nouvel instrument pour représenter la dispersion des revenus, le «boxplot» reproduit ici l'évaluation des données sexospécifiques des généralistes ainsi que des médecins effectuant des actes chirurgicaux. Le rec- 
tangle («box») indique la concentration de la moitié des revenus (deuxième et troisième quarts); ceci signifie que $50 \%$ du groupe considéré attestent de revenus situés dans cet intervalle. Le graphique permet, par exemple, les observations suivantes: un quart des médecins de premier recours gagnent au maximum 52100 francs, $50 \%$ de ce même groupe ( $1^{\text {er }}$ et $2^{\mathrm{e}}$ quarts) gagnent au maximum 100100 francs (= valeur centrale), la moitié moyenne ( $2^{\mathrm{e}}$ et $3^{\mathrm{e}}$ quarts) gagnent entre 52100 et 158750 francs, les revenus du dernier quart se situent entre 158750 et 1088000 francs. En comparaison: le revenu moyen de ce groupe (médecins de premier recours âgés de moins de 66 ans) se monte à 117750 francs, soit $17 \%$ environ au-dessus de la valeur centrale.

\section{B. Résultats: résumé}

\section{B.1 Généralités}

De 2005 à 2006, le renchérissement s'est élevé à $+1,05 \%$ selon l'indice suisse des prix à la consommation. Durant la même période, le revenu moyen a diminué en revanche de $-1,69 \%$ et le revenu central de
-0,99\% pour l'ensemble des médecins âgés de moins de 66 ans, ce qui correspond à une diminution réelle de $-2,71 \%$ pour le revenu moyen et de $-2,02 \%$ pour le revenu central. Par rapport à 2004, ce recul correspond à une diminution réelle de $-6,7 \%$ pour le revenu moyen et de $-6,0 \%$ pour le revenu central. Ainsi, la tendance décroissante des dernières années se poursuit à la fois pour le revenu moyen et pour le revenu central.

\section{B.2 Remarques préalables}

Les chiffres de 2005 ayant fait l'objet d'une nouvelle saisie et révisés diffèrent légèrement de ceux publiés dans l'étude de novembre 2008. Par conséquent, il est nécessaire de corriger la modification publiée à l'époque des valeurs moyennes des revenus des médecins entre 2004 et 2005 pour les groupes figurant ciaprès.

Le revenu moyen assujetti à l'AVS a diminué de 2,93\% (au lieu des 3,08\% publiés en 2008) pour la période de 2004 à 2005 suite à la réévaluation, le revenu central assujetti à l'AVS de 3,04\% (au lieu des 2,25\% publiés en 2008).

\section{Tableau 0a/06}

Pourcentage du nombre de médecins selon l'analyse des revenus (AR) par rapport au nombre de médecins selon la statistique médicale (SM), par canton (tous les médecins et selon le sexe), en 2006.

\begin{tabular}{|c|c|c|c|c|c|c|c|c|c|}
\hline & \multicolumn{3}{|c|}{ Tous les médecins } & \multicolumn{3}{|c|}{ Hommes } & \multicolumn{3}{|c|}{ Femmes } \\
\hline & AR & SM & $\%$ & AR & SM & $\%$ & AR & SM & $\%$ \\
\hline $\mathrm{ZH}$ & 2417 & 3610 & $67,0 \%$ & 1710 & 2504 & $68,3 \%$ & 707 & 1106 & $63,9 \%$ \\
\hline $\mathrm{BE}$ & 1886 & 2447 & $77,1 \%$ & 1475 & 1861 & $79,3 \%$ & 411 & 586 & $70,1 \%$ \\
\hline LU & 451 & 657 & $68,6 \%$ & 350 & 517 & $67,7 \%$ & 101 & 140 & $72,1 \%$ \\
\hline UR & 33 & 42 & $78,6 \%$ & 26 & 35 & $74,3 \%$ & 7 & 7 & $100,0 \%$ \\
\hline SZ & 174 & 197 & $88,3 \%$ & 136 & 149 & $91,3 \%$ & 38 & 48 & $79,2 \%$ \\
\hline UW & 85 & 93 & $91,4 \%$ & 71 & 79 & $89,9 \%$ & 14 & 14 & $100,0 \%$ \\
\hline GL & 47 & 58 & $81,0 \%$ & 40 & 46 & $87,0 \%$ & 7 & 12 & $58,3 \%$ \\
\hline ZG & 159 & 230 & $69,1 \%$ & 128 & 173 & $74,0 \%$ & 31 & 57 & $54,4 \%$ \\
\hline FR & 377 & 429 & $87,9 \%$ & 281 & 326 & $86,2 \%$ & 96 & 103 & $93,2 \%$ \\
\hline SO & 386 & 430 & $89,8 \%$ & 309 & 339 & $91,2 \%$ & 77 & 91 & $84,6 \%$ \\
\hline BS & 557 & 921 & $60,5 \%$ & 374 & 634 & $59,0 \%$ & 183 & 287 & $63,8 \%$ \\
\hline$B L$ & 554 & 614 & $90,2 \%$ & 410 & 444 & $92,3 \%$ & 144 & 170 & $84,7 \%$ \\
\hline $\mathrm{SH}$ & 146 & 157 & $93,0 \%$ & 114 & 119 & $95,8 \%$ & 32 & 38 & $84,2 \%$ \\
\hline AP & 107 & 117 & $91,5 \%$ & 96 & 94 & n.i. & 11 & 23 & $47,8 \%$ \\
\hline SG & 590 & 856 & $68,9 \%$ & 464 & 678 & $68,4 \%$ & 126 & 178 & $70,8 \%$ \\
\hline GR & 320 & 381 & $84,0 \%$ & 259 & 308 & $84,1 \%$ & 61 & 73 & $83,6 \%$ \\
\hline AG & 669 & 992 & $67,4 \%$ & 528 & 761 & $69,4 \%$ & 141 & 231 & $61,0 \%$ \\
\hline TG & 332 & 369 & $90,0 \%$ & 269 & 293 & $91,8 \%$ & 63 & 76 & $82,9 \%$ \\
\hline TI & 556 & 703 & $79,1 \%$ & 447 & 563 & $79,4 \%$ & 109 & 140 & $77,9 \%$ \\
\hline VD & 1120 & 1830 & $61,2 \%$ & 766 & 1273 & $60,2 \%$ & 354 & 557 & $63,6 \%$ \\
\hline VS & 357 & 513 & $69,6 \%$ & 290 & 407 & $71,3 \%$ & 67 & 106 & $63,2 \%$ \\
\hline NE & 206 & 363 & $56,7 \%$ & 153 & 271 & $56,5 \%$ & 53 & 92 & $57,6 \%$ \\
\hline GE & 254 & 1631 & $15,6 \%$ & 162 & 1066 & $15,2 \%$ & 92 & 565 & $16,3 \%$ \\
\hline J & 70 & 121 & $57,9 \%$ & 59 & 101 & $58,4 \%$ & 11 & 20 & $55,0 \%$ \\
\hline Suisse & 11853 & 17761 & $66,7 \%$ & 8917 & 13041 & $68,4 \%$ & 2936 & 4720 & $62,2 \%$ \\
\hline
\end{tabular}




\section{B.3 Aspects sexospécifiques}

La présente analyse comporte pour la première fois des évaluations complètes selon les sexes. Mesurée à sa population, la Suisse accuse un nombre important de médecins, tous sexes confondus; en comparaison internationale, en revanche, les chiffres absolus sont peu élevés dûs à sa plus faible population; l'évaluation des groupes exige donc une certaine prudence. Ceci vaut notamment pour les femmes médecins qui ne représentent qu'un quart du corps médical. Nous avons mentionné plus haut que nous n'analyserions des groupes qu'à partir de 20 valeurs individuelles pour des raisons de statistique mathématique. De nombreux sous-groupes (cantons, spécialisations, groupes d'âge) n'atteignant pas cette valeur minimale, il est donc difficile d'obtenir une analyse pertinente et fiable sur les revenus des femmes médecins.

La saisie des revenus des médecins indépendants, effectuée sans indication du degré d'occupation, rend la pertinence de conclusions en la matière encore plus difficile. Ainsi, les chiffres établis ne permettent pas de dire si les femmes médecins (cf. Tableau 3, p. 26s)

- gagnent près de 40 à $45 \%$ de moins pour le même travail

- prescrivent une médecine moins onéreuse

- travaillent 40 à $45 \%$ de moins

ou si une combinaison de ces trois énoncés explique ce frappant écart.

Le fait que les femmes sont plus nombreuses que les hommes à exercer la médecine à temps partiel permet néanmoins de conclure qu'une grande partie de cet écart important est due à cette situation.

La statistique médicale enregistre pour 2006 17761 médecins, dont 4720 de sexe féminin, soit une part de $26,6 \%$. En comparaison à cette moyenne, les taux les plus bas de femmes sont relevés dans les cantons des Grisons $(19,2 \%)$ et du Tessin $(19,9 \%)$, les taux les plus élevés dans les cantons de Bâle-Ville $(31,2 \%)$, de Zurich $(30,6 \%)$ et de Vaud $(30,4 \%)$.

Pour les spécialisations, la dispersion est nettement plus importante: alors que la proportion de femmes est inférieure à $5 \%$ en urologie, neurochirurgie et chirurgie orthopédique, elle dépasse $40 \%$ en dermatologie et en pédiatrie. La Psychiatrie pour enfants et adolescents occupe la tête de liste avec une proportion de presque $60 \%$ de femmes, plus du double de la valeur moyenne. Les chiffres des médecins répertoriés par la caisse de compensation medisuisse attestent une situation similaire, même si les facteurs de dispersion y sont en partie plus importants dus à la population analysée plus restreinte.

Les séries de chiffres qui ne visent pas la comparaison directe, mais qui montrent, à l'intérieur du même sexe, des schémas étroitement liés à la structure des revenus sont particulièrement intéressantes pour la comparaison des sexes. Ainsi par exemple, il n'est pas surprenant que $2 \%$ seulement des femmes médecins font état d'un revenu supérieur à 200000 francs contre $50 \%$ chez les hommes -, puisqu'il y a appa- remment une concentration des petits revenus chez les femmes. La répartition par spécialisation a déjà été mentionnée et révèle une nette préférence des femmes pour la médecine non opératoire. En médecine générale, les femmes représentent une part de $20 \%$ à peine; ceci pourrait s'expliquer par le fait qu'un cabinet de médecine générale rend plus difficile l'exercice d'une activité professionnelle partielle qu'un cabinet psychiatrique par exemple.

Un analyse du temps de travail est la seule manière d'obtenir des résultats pertinents sur les différentes situations financières des deux sexes pour la profession de médecin. Hormis les inclinations personnelles, le choix d'une spécialisation repose probablement sur la possibilité pour les femmes d'exercer leur activité professionnelle à temps partiel.

\section{B.4 Evolution des revenus}

Entre 2005 et 2006, la hausse du renchérissement a atteint $+1,05 \%$. Durant la même période, le revenu moyen des médecins (revenu assujetti à l'AVS) a diminué de 212345 à 208755 francs (soit une diminution de $-1,69 \%$ ) et le revenu central des médecins de 172500 à 170800 francs (soit une diminution de 0,99\%).

Entre 2005 et 2006 , le revenu moyen a nettement diminué pour tous les médecins, tandis que le renchérissement a connu une légère hausse. Par conséquent, on constate une diminution réelle de $-2,71$ à $-5,18 \%$ des revenus. Pour le revenu central des médecins, on enregistre une diminution réelle de $-0,67$ à $-2,64 \%$.

Chez l'ensemble des médecins indépendants âgés de moins de 66 ans, l'évolution des revenus de 1971/72 à 2006 révèle une augmentation nominale du revenu moyen de $+61,4 \%$ et du revenu central de $+48,6 \%$. Si l'on tient compte toutefois du renchérissement de $+163,2 \%$ observé durant la même période, on constate une perte réelle de $-38,7 \%$ pour le revenu moyen et de $-43 \%$ pour le revenu central des médecins indépendants âgés de moins de 66 ans.

Mais si l'on indexe l'évolution des revenus des médecins indépendants âgés de moins de 66 ans entre 1971/72 et 2006 sur l'évolution des salaires des travailleurs, c'est-à-dire en comparant nominalement l'évolution du revenu des médecins à celle des salaires (ou des salaires des secteurs II et III), on constate une perte relative respective de $-52,7$ et $-51,3 \%$ pour le revenu moyen des médecins indépendants âgés de moins de 66 ans.

Les différences entre les revenus moyens par cantons et spécialisations restent importantes malgré l'introduction du TARMED; jusqu'en 2004, ces différences avaient plutôt tendance à diminuer chaque année; elles ont à nouveau augmenté pour la première fois en 2005 et se sont creusées encore davantage en 2006 notamment entre les spécialisations (voir également chapitre C.18, page $77 \mathrm{~s})$.

Les moyennes par canton se situent, pour les médecins indépendants âgés de moins de 66 ans, entre 
Tableau 0b/06

Pourcentage du nombre de médecins selon l'analyse des revenus (AR) par rapport au nombre de médecins selon la statistique médicale (SM), par spécialisation (tous les médecins et selon le sexe), en 2006.

\begin{tabular}{|c|c|c|c|c|c|c|c|c|c|}
\hline \multirow[b]{2}{*}{ Spécialisation } & \multicolumn{3}{|c|}{ Tous les médecins } & \multicolumn{3}{|c|}{ Hommes } & \multicolumn{3}{|c|}{ Femmes } \\
\hline & AR & SM & $\%$ & AR & SM & $\%$ & AR & SM & $\%$ \\
\hline Anesthésiologie & 269 & 1010 & $26,6 \%$ & 180 & 665 & $27,1 \%$ & 89 & 345 & $25,8 \%$ \\
\hline Chirurgie & 566 & 1087 & $52,1 \%$ & 516 & 975 & $52,9 \%$ & 50 & 112 & $44,6 \%$ \\
\hline Dermatologie & 248 & 286 & $86,7 \%$ & 155 & 168 & $92,3 \%$ & 93 & 118 & $78,8 \%$ \\
\hline Gynécologie & 757 & 1029 & $73,6 \%$ & 465 & 649 & $71,6 \%$ & 292 & 380 & $76,8 \%$ \\
\hline Médecine interne & 2288 & 4413 & $51,8 \%$ & 1882 & 3442 & $54,7 \%$ & 406 & 971 & $41,8 \%$ \\
\hline Neurochirurgie & 39 & 64 & $60,9 \%$ & 36 & 62 & $58,1 \%$ & 3 & 2 & n.i. \\
\hline Neurologie & 175 & 275 & $63,6 \%$ & 140 & 214 & $65,4 \%$ & 35 & 61 & $57,4 \%$ \\
\hline Ophtalmologie & 427 & 606 & $70,5 \%$ & 281 & 386 & $72,8 \%$ & 146 & 220 & $66,4 \%$ \\
\hline Orthopédie & 295 & 502 & $58,8 \%$ & 284 & 481 & $59,0 \%$ & 11 & 21 & $52,4 \%$ \\
\hline ORL & 215 & 336 & $64,0 \%$ & 185 & 276 & $67,0 \%$ & 30 & 60 & $50,0 \%$ \\
\hline Pédiatrie & 600 & 957 & $62,7 \%$ & 370 & 531 & $69,7 \%$ & 230 & 426 & $54,0 \%$ \\
\hline Pathologie & 10 & 125 & $8,0 \%$ & 10 & 71 & $14,1 \%$ & 0 & 54 & $0,0 \%$ \\
\hline Médecine physique & 158 & 121 & n.i. & 132 & 91 & n.i. & 26 & 30 & $86,7 \%$ \\
\hline Psychiatrie & 1604 & 2184 & $73,4 \%$ & 978 & 1352 & $72,3 \%$ & 626 & 832 & $75,2 \%$ \\
\hline Psychiatrie pour enfants & 227 & 377 & $60,2 \%$ & 94 & 148 & $63,5 \%$ & 133 & 229 & $58,1 \%$ \\
\hline Radiologie* & 144 & 457 & $31,5 \%$ & 116 & 353 & $32,9 \%$ & 28 & 104 & $26,9 \%$ \\
\hline Médecine tropicale & 3 & 33 & $9,1 \%$ & 3 & 29 & $10,3 \%$ & 0 & 4 & $0,0 \%$ \\
\hline Urologie & 112 & 175 & $64,0 \%$ & 109 & 171 & $63,7 \%$ & 3 & 4 & $75,0 \%$ \\
\hline Médecine générale & 3716 & 3724 & $99,8 \%$ & 2981 & 2977 & n.i. & 735 & 747 & $98,4 \%$ \\
\hline Toutes les spécialisations & 11853 & 17761 & $66,7 \%$ & 8917 & 13041 & $68,4 \%$ & 2936 & 4720 & $62,2 \%$ \\
\hline
\end{tabular}

termes en grasse $=$ aucune évaluation ( ${ }^{*}$ sexospécifique)

Tableau 5/06

Revenus assujettis à l'AVS de tous les médecins indépendants (H et F), valeurs moyenne et centrale par canton, médecins âgés de moins de 66 ans, en 2006 et modifications.

\begin{tabular}{|c|c|c|c|c|c|c|c|c|}
\hline 2006 & Nombre & Valeur moyenne & Valeur centrale & Maximum & Indice VM & Indice VC & VM +/- & VC +l- \\
\hline $\mathrm{ZH}$ & 2246 & 198275 & 154850 & 2188900 & $95,0 \%$ & $90,7 \%$ & $-3,1 \%$ & $-0,2 \%$ \\
\hline $\mathrm{BE}$ & 1772 & 207388 & 162500 & 1900300 & $99,3 \%$ & $95,1 \%$ & $0,7 \%$ & $0,7 \%$ \\
\hline LU & 430 & 252223 & 224000 & 1109600 & $120,8 \%$ & $131,1 \%$ & $-1,7 \%$ & $1,5 \%$ \\
\hline UR & 32 & 221597 & 203400 & 516400 & $106,2 \%$ & $119,1 \%$ & $3,5 \%$ & $-1,0 \%$ \\
\hline$S Z$ & 164 & 250837 & 217200 & 979800 & $120,2 \%$ & $127,2 \%$ & $-4,9 \%$ & $-12,2 \%$ \\
\hline UW & 84 & 214389 & 183400 & 694500 & $102,7 \%$ & $107,4 \%$ & $-0,9 \%$ & $-8,0 \%$ \\
\hline$G L$ & 44 & 271791 & 269700 & 842500 & $130,2 \%$ & $157,9 \%$ & $2,4 \%$ & $0,2 \%$ \\
\hline$Z G$ & 149 & 217059 & 189100 & 772600 & $104,0 \%$ & $110,7 \%$ & $0,3 \%$ & $-5,3 \%$ \\
\hline FR & 354 & 222832 & 193250 & 1036200 & $106,7 \%$ & $113,1 \%$ & $-2,1 \%$ & $0,8 \%$ \\
\hline SO & 371 & 215385 & 181400 & 3613200 & $103,2 \%$ & $106,2 \%$ & $-3,6 \%$ & $0,0 \%$ \\
\hline BS & 507 & 170282 & 136600 & 1345700 & $81,6 \%$ & $80,0 \%$ & $-1,1 \%$ & $-1,8 \%$ \\
\hline$B L$ & 514 & 230056 & 185050 & 2651800 & $110,2 \%$ & $108,3 \%$ & $3,3 \%$ & $4,9 \%$ \\
\hline $\mathrm{SH}$ & 139 & 203840 & 159100 & 1360300 & $97,6 \%$ & $93,1 \%$ & $-4,4 \%$ & $1,5 \%$ \\
\hline AP & 99 & 205368 & 188300 & 676300 & $98,4 \%$ & $110,2 \%$ & $-12,0 \%$ & $-2,7 \%$ \\
\hline SG & 552 & 251432 & 221800 & 1939600 & $120,4 \%$ & $129,9 \%$ & $3,4 \%$ & $2,1 \%$ \\
\hline GR & 301 & 178731 & 145000 & 880600 & $85,6 \%$ & $84,9 \%$ & $-8,0 \%$ & $-14,1 \%$ \\
\hline$A G$ & 624 & 225264 & 192450 & 1680900 & $107,9 \%$ & $112,7 \%$ & $-2,0 \%$ & $-0,7 \%$ \\
\hline TG & 322 & 224878 & 191050 & 2269800 & $107,7 \%$ & $111,9 \%$ & $-0,9 \%$ & $-6,3 \%$ \\
\hline TI & 510 & 220290 & 174800 & 1261900 & $105,5 \%$ & $102,3 \%$ & $-3,1 \%$ & $-6,8 \%$ \\
\hline VD & 1071 & 198948 & 160100 & 1136900 & $95,3 \%$ & $93,7 \%$ & $-5,2 \%$ & $-9,9 \%$ \\
\hline VS & 339 & 181072 & 162300 & 816800 & $86,7 \%$ & $95,0 \%$ & $-1,2 \%$ & $-4,6 \%$ \\
\hline NE & 196 & 154928 & 140900 & 530600 & $74,2 \%$ & $82,5 \%$ & $5,6 \%$ & $6,1 \%$ \\
\hline GE & 231 & n.i. & n.i. & n.i. & n.i. & n.i. & n.i. & n.i. \\
\hline JU & 62 & 169442 & 174800 & 403000 & $81,2 \%$ & $102,3 \%$ & $6,7 \%$ & $10,4 \%$ \\
\hline Suisse & 11113 & 208755 & 170800 & 3613200 & $100,0 \%$ & $100,0 \%$ & $-1,7 \%$ & $-1,0 \%$ \\
\hline
\end{tabular}


69,1 et $125,0 \%$ (facteur 1,81 ) de la valeur moyenne suisse pour 2005 et entre 74,2 et $130,2 \%$ (facteur 1,75 ) de la valeur moyenne suisse pour 2006.

Les chiffres de la dispersion des revenus moyens par spécialisation remontent jusqu'à l'exercice 2004, ils augmentent légèrement en 2005: chez les médecins indépendants âgés de moins de 66 ans, les moyennes de revenus par spécialisation se situent entre 54,9 et $188,9 \%$ (facteur 3,44 ) en 2005 , entre 52,8 et $205,0 \%$ (facteur 3,88 ) de la valeur moyenne suisse en 2006.

La différence entre les médecins effectuant des actes chirurgicaux et ceux qui n'en effectuent pas reste importante. Comme le revenu des premiers résulte essentiellement du tarif privé, il est, pour les médecins âgés de moins de 66 ans, en moyenne de $47 \%$ pour

\section{Tableau 9/06}

Revenus assujettis à l'AVS de tous les médecins indépendants (H et F), valeurs moyenne et centrale par spécialisation, médecins âgés de moins de 66 ans, en 2006 et modifications.

\begin{tabular}{|c|c|c|c|c|c|c|c|c|}
\hline 2006 & Nombre & Valeur moyenne & Valeur centrale & Maximum & Indice VM & Indice VC & VM +/- & VC +/- \\
\hline Anesthésiologie & 254 & 268025 & 250050 & 999000 & $128,4 \%$ & $146,4 \%$ & $1,4 \%$ & $17,0 \%$ \\
\hline Chirurgie & 500 & 243227 & 166400 & 1324300 & $116,5 \%$ & $97,4 \%$ & $-1,0 \%$ & $-0,4 \%$ \\
\hline Dermatologie & 236 & 240589 & 203050 & 2188900 & $115,2 \%$ & $118,9 \%$ & $2,1 \%$ & $-1,0 \%$ \\
\hline Gynécologie & 706 & 241539 & 208550 & 1545800 & $115,7 \%$ & $122,1 \%$ & $-5,2 \%$ & $-3,0 \%$ \\
\hline Médecine interne & 2154 & 222231 & 186000 & 1969700 & $106,5 \%$ & $108,9 \%$ & $-0,9 \%$ & $-0,4 \%$ \\
\hline Neurochirurgie & 35 & 427974 & 354000 & 1507100 & $205,0 \%$ & $207,3 \%$ & $6,7 \%$ & $11,7 \%$ \\
\hline Neurologie & 156 & 159800 & 138450 & 621800 & $76,5 \%$ & $81,1 \%$ & $-3,7 \%$ & $-6,4 \%$ \\
\hline Ophtalmologie & 394 & 334759 & 288150 & 2269800 & $160,4 \%$ & $168,7 \%$ & $1,7 \%$ & $6,8 \%$ \\
\hline Orthopédie & 278 & 286817 & 246600 & 1566500 & $137,4 \%$ & $144,4 \%$ & $-5,2 \%$ & $-6,9 \%$ \\
\hline ORL & 207 & 289604 & 239500 & 912100 & $138,7 \%$ & $140,2 \%$ & $-2,1 \%$ & $-0,7 \%$ \\
\hline Pédiatrie & 549 & 169440 & 154500 & 693200 & $81,2 \%$ & $90,5 \%$ & $-0,6 \%$ & $3,8 \%$ \\
\hline Médecine physique & 148 & 211386 & 203100 & 596900 & $101,3 \%$ & $118,9 \%$ & $1,6 \%$ & $9,4 \%$ \\
\hline Psychiatrie & 1484 & 127775 & 118150 & 788600 & $61,2 \%$ & $69,2 \%$ & $-2,4 \%$ & $-3,2 \%$ \\
\hline Psychiatrie pour enfants & 212 & 110208 & 111000 & 365900 & $52,8 \%$ & $65,0 \%$ & $-5,4 \%$ & $1,3 \%$ \\
\hline Radiologie & 139 & 339369 & 253100 & 2651800 & $162,6 \%$ & $148,2 \%$ & $-4,7 \%$ & $-7,8 \%$ \\
\hline Urologie & 107 & 312075 & 263100 & 1392200 & $149,5 \%$ & $154,0 \%$ & $-0,5 \%$ & $1,8 \%$ \\
\hline Spécialistes & 7571 & 215933 & 166700 & 3613200 & $103,4 \%$ & $97,6 \%$ & $-1,8 \%$ & $-1,9 \%$ \\
\hline Médecine générale & 3542 & 193412 & 175950 & 1680900 & $92,7 \%$ & $103,0 \%$ & $-1,5 \%$ & $-1,2 \%$ \\
\hline Tous les médecins & 11113 & 208755 & 170800 & 3613200 & $100,0 \%$ & $100,0 \%$ & $-1,7 \%$ & $-1,0 \%$ \\
\hline
\end{tabular}

Tableau 29

Extrait de la liste de la valeur du point tarifaire TARMED LAMal du 1er janvier 2004 (entrée en vigueur du TARMED) au 1er janvier 2008 (en centimes).

\begin{tabular}{|c|c|c|c|c|c|c|c|}
\hline & 1.1 .2004 & 1.9. 2004 & 1.1.2005 & 1.7.2005 & 1. 1.2006 & 1.1.2007 & 1. 1.2008 \\
\hline $\mathrm{ZH}$ & 97 & 95 & 92 & 90 & 91 & 90 & 89 \\
\hline $\mathrm{BE}$ & 89 & 86 & 84 & 86 & 87 & 86 & 86 \\
\hline FR & 91 & 91 & 88 & 86 & 89 & 92 & 91 \\
\hline SO & 87 & 84 & 84 & 81 & 84 & 84 & 84 \\
\hline BS & 93 & 93 & 89 & 87 & 88 & 89 & 89 \\
\hline$B L$ & 97 & 97 & 92 & 95 & 96 & 93 & 94 \\
\hline GR & 84 & 78 & 78 & 78 & 78 & 80 & 80 \\
\hline $\mathrm{AG}$ & 89 & 89 & 87 & 92 & 90 & 89 & 89 \\
\hline TI & 91 & 97 & 97 & 97 & 97 & 97 & 96 \\
\hline VD & 92 & 96 & 98 & 98 & 98 & 98 & 98 \\
\hline VS & 78 & 78 & 78 & 78 & 78 & 80 & 81 \\
\hline NE & 94 & 89 & 89 & 89 & 91 & 92 & 92 \\
\hline GE & 98 & 98 & 98 & 98 & 98 & 96 & 96 \\
\hline JU & 95 & 95 & 97 & 99 & 99 & 99 & 99 \\
\hline $\mathrm{CH}$ centrale & 86 & 83 & 80 & 80 & 80 & 86 & 86 \\
\hline $\mathrm{CH}$ orientale & 85 & 82 & 82 & 84 & 84 & 82 & 82 \\
\hline Moyenne & 90,38 & 89,44 & 88,31 & 88,63 & 89,25 & 89,56 & 89,50 \\
\hline
\end{tabular}


2005 et de $46 \%$ pour 2006 supérieur à celui des médecins n'effectuant pas d'actes chirurgicaux.

La comparaison entre les valeurs statistiques des médecins de premier recours (internistes, pédiatres et généralistes) et celles des médecins effectuant des actes chirurgicaux (chirurgiens, gynécologues, ophtalmologues, orthopédistes et urologues) montre dans le Tableau 20 de la page 65 que non seulement les valeurs centrales se différencient de 26 à $28 \%$, mais aussi que les quantiles (moitiés, tiers, quarts) se trouvent, pour les médecins effectuant des actes chirurgicaux, à des niveaux nettement supérieurs. Ainsi par exemple, le facteur du deuxième tercile des médecins de premier recours par rapport aux médecins effectuant des actes chirurgicaux s'élève respectivement à 1:1,39 en 2005 et $1: 1,41$ en 2006 , pour le troisième quartile à $1: 1,47$ en 2005 et 1:1,46 en 2006 .

Les valeurs centrales publiées par canton sont à peine supérieures et celles publiées par spécialisation à peine inférieures aux valeurs moyennes.

Les valeurs centrales par canton se situent, pour les médecins indépendants âgés de moins de 66 ans, entre 77,0 et $156,0 \%$ (facteur 2,03 ) de la valeur centrale suisse en 2005 et entre 78,5 et $157,9 \%$ (facteur 2,01) de la valeur centrale suisse en 2006.

Les valeurs centrales par spécialisation se situent, pour les médecins indépendants âgés de moins de 66 ans, entre 63,5 et 183,8\% (facteur 2,89 ) de la valeur centrale suisse en 2005 et entre 65,0 et $207,3 \%$ (facteur 3,19 ) de la valeur centrale suisse en 2006.

La masse salariale augmente avec l'accroissement du revenu moyen.

\section{B.5 Répartition fréquentielle des revenus}

La répartition fréquentielle des revenus reste dans le champ inférieur de manière semblable aux analyses précédentes: la moitié des médecins indépen-

\section{Figure 36 (Boxplot)}

Revenus assujettis à l'AVS des médecins de premier recours et des médecins effectuant des actes chirurgicaux, en 2006 selon le sexe (cf. tableau 20, p. 65).

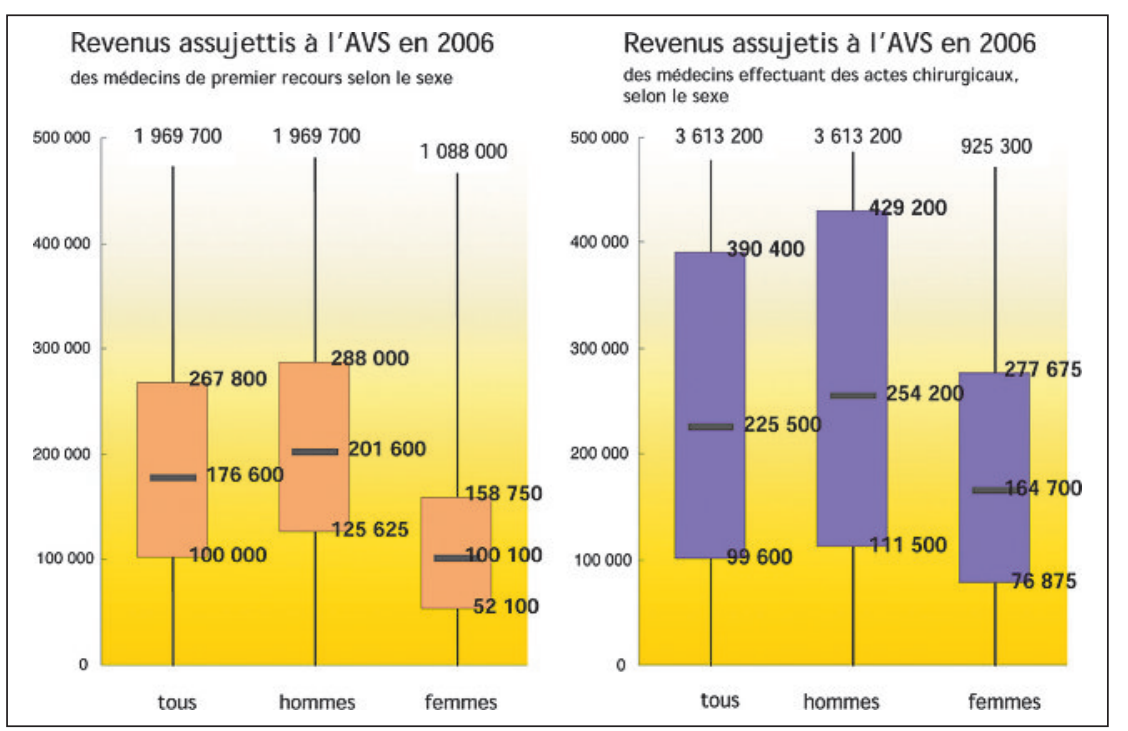

dants âgés de moins de 66 ans atteste en 2006 (2005) un revenu assujetti à l'AVS inférieur à 170800 francs (172500 francs). Selon l'étude de la SUVA/SSO, ce revenu correspond à un salaire d'employé de 120500 francs (121 700 francs). Selon l'étude de la SCTM, ce chiffre correspond à un salaire brut d'employé de 145000 francs (146400 francs). Cf. également le chapitre B.3.4 à la page 9.

La moitié moyenne des médecins (deuxième et troisième quarts) indépendants âgés de moins de 66 ans atteste en 2006 (2005) un revenu assujetti à l'AVS se situant entre 90900 et 277000 francs (92400 et 279000 francs).

Un quart des médecins indépendants âgés de moins de 66 ans atteste en 2006 (2005) un revenu assujetti à l'AVS inférieur à 90900 francs (92400 francs).

Un quart des médecins indépendants âgés de moins de 66 ans atteste en 2006 (2005) un revenu assujetti à l'AVS supérieur à 277000 francs (279000 francs).

Le tiers moyen des médecins indépendants âgés de moins de 66 ans atteste en 2006 (2005) un revenu assujetti à l'AVS se situant entre 118900 et 233700 francs (119800 et 237600 francs).

Un tiers des médecins indépendants âgés de moins de 66 ans atteste en 2006 (2005) un revenu assujetti à l'AVS inférieur à 118900 francs (119800 francs).

Un tiers des médecins indépendants âgés de moins de 66 ans atteste en 2006 (2005) un revenu assujetti à l'AVS supérieur à 233700 francs (237 600 francs).

Le fait de ne pas pouvoir mettre le revenu des médecins en relation avec la quantité et de ne pouvoir s'appuyer que partiellement sur la qualité du travail fourni est l'une des difficultés fondamentales rencontrées dans l'évaluation de ces revenus.

\section{B.6 Tendances}

Entre 2005 et 2006, les revenus des médecins ont à nouveau diminué face à une augmentation minime de l'indice du coût de la vie. En d'autres termes, on constate une baisse réelle des revenus durant cette période.

Les facteurs entre le revenu le plus élevé et le revenu le plus bas par canton et par spécialisation ont légèrement jusqu'à nettement diminué jusqu'en 2004, année à partir de laquelle on constate une nouvelle augmentation marquée du revenu, notamment par spécialisation.

Les relevés des prochaines années continueront de former la base pour analyser les répercussions du tarif TARMED au moyen des facteurs définis au chapitre C.18. à la page 77s, lequel a aussi pour but de revaloriser les prestations médicales intellectuelles au détriment des prestations de technique opératoire.

L'augmentation des revenus des médecins en 2004 est à mettre en relation avec l'introduction du tarif TARMED. Elle était attendue par la Commission paritaire Médecins/Caisses-maladie sur la base de l'observation de la neutralité des coûts. En 2004 et 2005, la valeur du point tarifaire du tarif TARMED a baissé 
à plusieurs reprises, laissant ainsi présager la baisse constatée des revenus des médecins en 2005. Malgré la hausse du point tarifaire au second semestre 2005 et en 2006, on observe un nouveau recul des revenus des médecins.

B.6.1 Influence de la valeur du point tarifaire TARMED LAMal sur le revenu des médecins

Comme indiqué plus haut, la baisse des revenus des médecins entre 2004 et 2005 était attendue depuis un certain temps déjà, suite à la baisse répétée de la valeur du point tarifaire TARMED après le $1^{\text {er }}$ janvier 2004 par la Commission paritaire de la neutralité des coûts Médecins et Caisses-maladie. Dans le Tableau 29 ciaprès, des extraits de la valeur du point tarifaire TARMED prévue pour la LAMal sont indiqués pour la période allant du $1^{\text {er }}$ janvier 2004 (entrée en vigueur du TARMED) au $1^{\text {er }}$ janvier 2009.

\section{Tableau 23c}

Indices des revenus assujettis à l'AVS des médecins indépendants de Suisse (jusqu'en 1975/76 pour les médecins âgés de moins de 63 ans, dès 1977/78 pour les médecins âgés de moins de 66 ans), de 1971/72 à 2006. Troisième partie 1995/96 à 2006. Toutes les séries d'indices sont calculées sur la base des années $1971 / 72=100,0$.

Evolution des revenus: indices (uniquement pour médecins âgées de moins de 66 ans)

$\begin{array}{lrrrrrrrrr} & 1995 / 96 & 1997 / 98 & 2001 & 2002 & 2003 & 2004 & 2005 & 2006 \\ \text { Prix à la consommation } & 241,6 & 243,8 & 252,1 & 253,7 & 255,4 & 257,4 \\ \text { Modification } & & +0,9 \% & & +3,4 \% & & +0,6 \% & +0,7 \% & +0,8 \% & +1,2 \%\end{array}$

Revenus assujettis à l'AVS

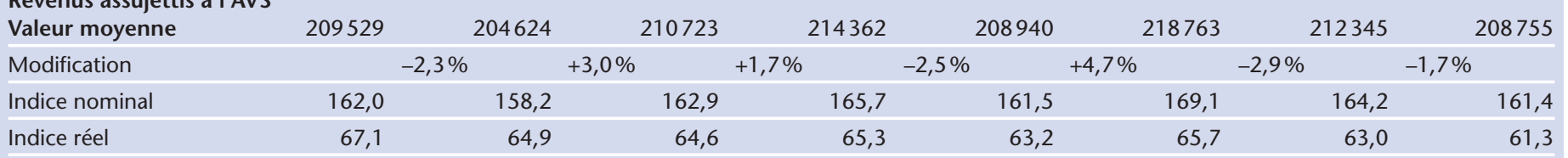

Revenu assujetti à I'AVS

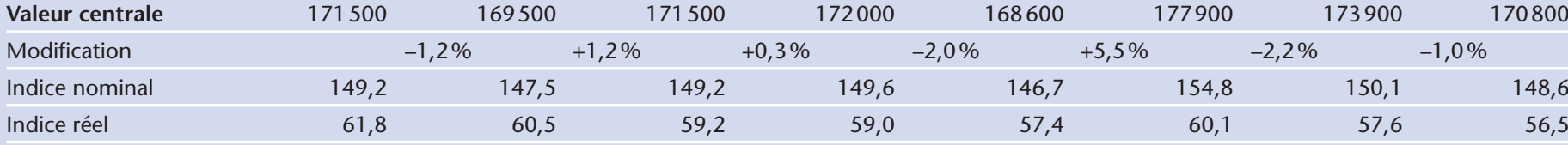

Salaires des travailleurs

$\begin{array}{llllllr}\text { Indice nominal } & 303,7 & 307,3 & 319,8 & 325,8 & 329,6 & 332,1 \\ \text { Indice réel } & 125,7 & 126,1 & 126,9 & 128,4 & 129,1\end{array}$

Salaires secteur II (production) Base de l'indice 1971/72 = 100,0 (Base 1993 nominal = 294,2, réel = 125,1)

$\begin{array}{llllllll}\text { Indice nominal } & 304,5 & 308,0 & 322,1 & 324,4 & 331,3 & 333,3 & 337,4 \\ \text { Indice réel } & 125,6 & 125,9 & 127,2 & 128,6 & 129,2 & 129,0\end{array}$

Salaires des employés

$\begin{array}{lllllll}\text { Indice nominal } & 293,1 & 298,2 & 312,6 & 317,7 & 322,9 & 326,5 \\ \text { Indice réel } & 121,3 & 122,3 & 124,0 & 125,2 & 126,4\end{array}$

Salaires secteur III (prestations de services) Base de l'indice 1971/72=100,0 (Base 1993 nominal = 283,0, réel = 120,3)

\begin{tabular}{|c|c|c|c|c|c|c|c|c|}
\hline Indice nominal & 292,6 & 297,4 & 303,4 & 316,1 & 320,9 & 324,9 & 327,4 & 331,4 \\
\hline Indice réel & 120,6 & 121,5 & 122,6 & 124,0 & 125,2 & 125,7 & 125,2 & 125,5 \\
\hline
\end{tabular}

Revenus assujettis à l'AVS Valeur moyenne nominale indexée sur l'indice de base 1971/72=100,0

\begin{tabular}{|c|c|c|c|c|c|c|c|c|}
\hline Salaires secteur II & 53,2 & 51,4 & 50,6 & 51,1 & 48,7 & 50,7 & 48,7 & 47,3 \\
\hline Salaires secteur III & 55,4 & 53,2 & 53,7 & 52,4 & 50,3 & 52,0 & 50,2 & 48,7 \\
\hline
\end{tabular}

Modifications de la valeur centrale, nominale

\begin{tabular}{|c|c|c|c|c|c|c|}
\hline & $1995 / 96$ & $1997 / 98$ & 2001 & 2002 & 2004 & 2006 \\
\hline Revenus assujettis à I'AVS & $+0,5 \%$ & $-1,2 \%$ & $+1,2 \%$ & $+0,3 \%$ & $+3,4 \%$ & $-4,0 \%$ \\
\hline & 1996 & 1998 & 2000 & 2002 & 2004 & 2006 \\
\hline Salaires secteur II & $+3,4 \%$ & $+1,7 \%$ & $+2,2 \%$ & $+5,1 \%$ & $+2,0 \%$ & $+2,0 \%$ \\
\hline Salaires secteur III & $+2,9 \%$ & $+2,8 \%$ & $+2,3 \%$ & $+3,0 \%$ & $+2,8 \%$ & $+2,7 \%$ \\
\hline
\end{tabular}




\section{Figure 13}

Evolution de la valeur moyenne des revenus assujettis à I'AVS des médecins indépendants de Suisse âgés de moins de 66 ou 63 ans (nominal), de 1971/72 à 2006 (cf. Tableau 23, p. 67ss).

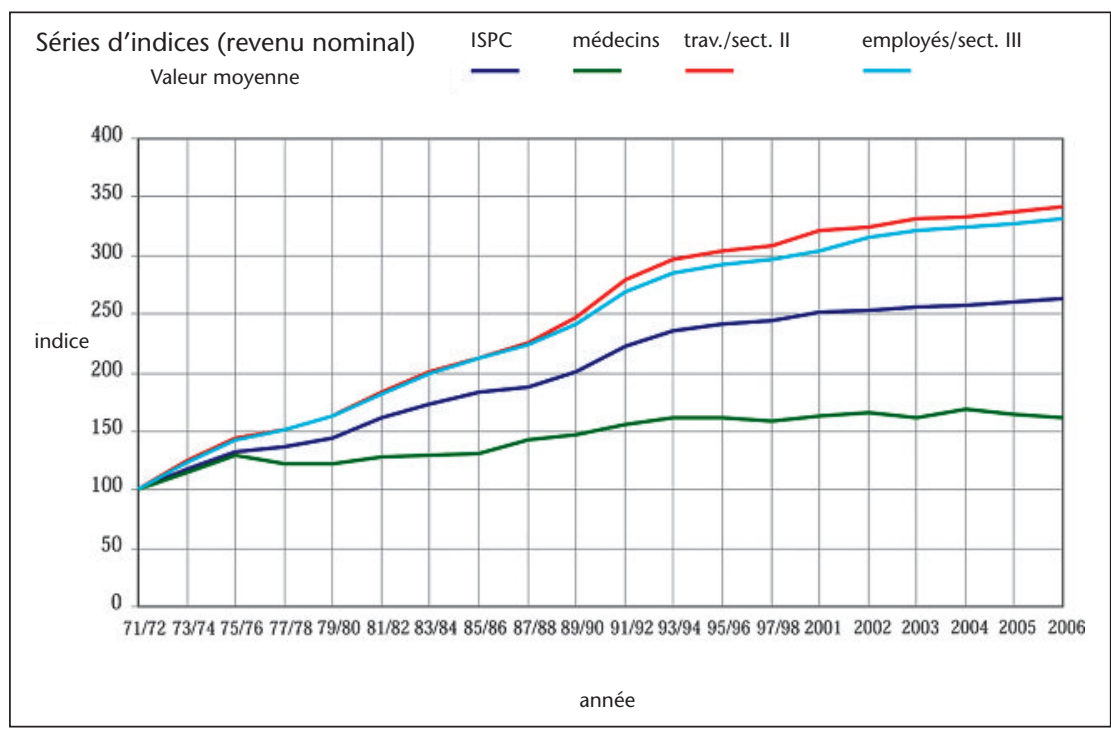

Figure 14

Evolution de la valeur moyenne des revenus assujettis à l'AVS des médecins indépendants de Suisse âgés de moins de 66 ou 63 ans (réel), de 1971/72 à 2006 (cf. Tableau 23, p. 67ss).

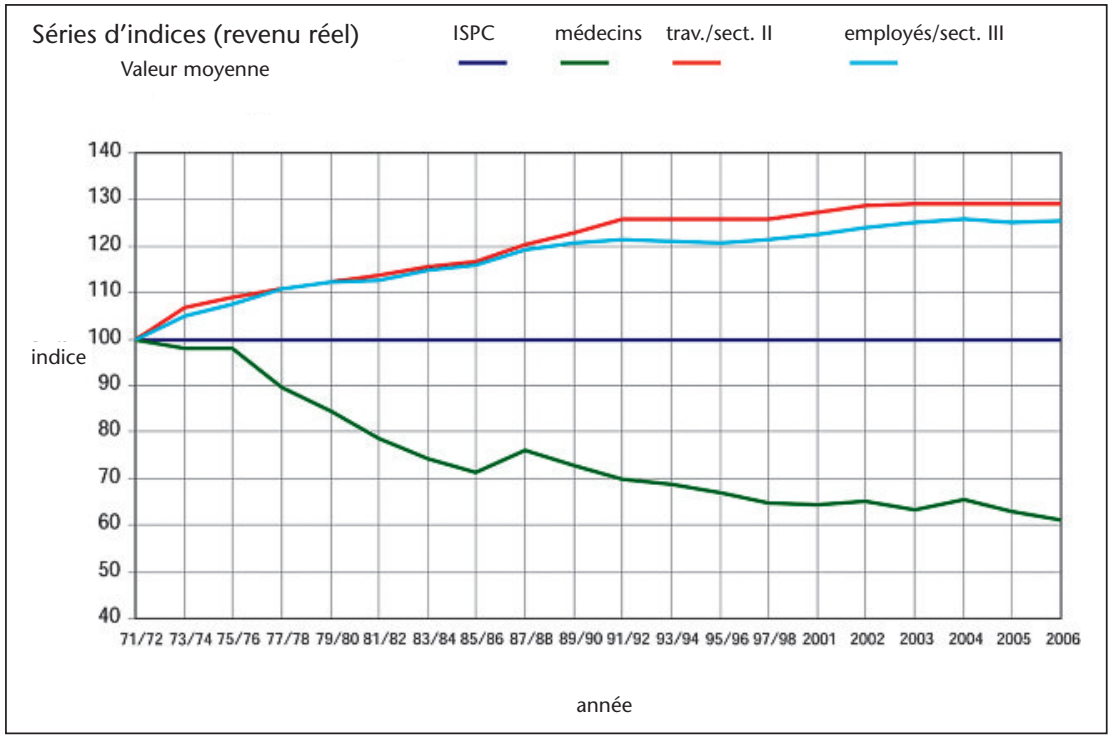

Avant de signer les conventions relatives au TARMED, la FMH et le Concordat des assureurs-maladie suisse ont fixé de manière détaillée dans des conventions spéciales le fonctionnement de la Commission paritaire de la neutralité des coûts et le recensement des données de base statistiques nécessaires provenant directement de la facturation des médecins. La baisse ou la hausse de la valeur du point tarifaire est devenue une simple tâche de statistique mathématique qui a permis d'introduire le nouveau tarif sans provoquer d'importantes hausses ou baisses du volume global des honoraires.

Le léger dépassement vers le haut du volume des honoraires facturés a été compensé les années suivantes par une baisse nettement plus importante de la valeur du point tarifaire. La simple hausse de la valeur du point tarifaire entre 2005 et 2006 a plus que dépassé l'un des objectifs déclarés du TARMED, à savoir éviter l'augmentation des revenus bruts des médecins. Une légère augmentation des revenus des médecins en 2006 suite à la hausse de la valeur du point tarifaire, comme l'avait supposée la dernière analyse, ne s'est donc pas confirmée. Malgré la hausse de la valeur du point tarifaire d'environ 1\% entre 2005 et 2006, les revenus des médecins indépendants ont reculé. Il sera donc intéressant d'observer les répercussions qu'aura eu la nouvelle hausse de la valeur de point tarifaire de $0,3 \%$ en 2007.

B.6.2 Evolution des revenus de 1971/72 à 2006

Les chiffres révisés concernant les revenus de 2005 et les nouveaux chiffres concernant les revenus de 2006 permettent maintenant de suivre correctement l'évolution de revenu moyen et central assujetti à l'AVS des médecins indépendants de Suisse sur trois décennies au moins.

Les graphiques 13 et 14 (annexe page 101) présentent les séries d'indices pour le revenu moyen assujetti à l'AVS (uniquement médecins âgés de moins de 66 ans), aussi bien sous forme nominale que réelle (c'est-à-dire renchérissement inclus). Ces graphiques montrent clairement que l'écart entre le revenu de la population active et celui des médecins indépendants arapidement que dans les années 70 et 80. 